\title{
Psychopathie und antisoziale Persönlichkeitsstörungen im Spannungsfeld zwischen Pathologie und Dissozialität
}

\author{
Henning Saß $\aleph^{1}$ Elmar Habermeyer ${ }^{2}$ \\ ๑) Springer-Verlag GmbH Deutschland, ein Teil von Springer Nature 2018

\section{Psychopathy and antisocial personality disorders in the field of tension between pathology and dissociality}

Die schon im allgemeinen Sprachgebrauch vieldeutigen, einer Stigmatisierung Vorschub leistenden Begriffe von Persönlichkeitsstörung und Psychopathie begegnen in der forensischen Anwendung vermehrten Schwierigkeiten. Zwar enthalten die modernen Klassifikationssysteme verbindliche diagnostische Algorithmen mit klaren Ein- und Ausschlusskriterien, doch liegt jenseits der Pseudoexaktheit solcher Formulierungen das Kernproblem weiterhin in einer konzeptionellen Differenzierung von Charakterpathologie und abweichendem Verhalten. Bei vielen Straftätern finden sich auf der einen Seite schwierige, zu sozialen Konflikten disponierende Persönlichkeitsmerkmale und auf der anderen Seite mehr oder weniger hartnäckige Neigungen zur Verletzung rechtlicher Normen. Unerlässlich für die Bearbeitung der forensischen Fragestellungen ist deshalb eine klare Unterscheidung zwischen psychopathologisch bedeutsamen Auffälligkeiten der Persönlichkeit und bloßer Dissozialität. Dem steht allerdings seit Beginn der psychiatrischen Beschreibungen auffälliger Persönlichkeiten eine hartnäckige Tendenz zur Verquickung der Konzepte von abnormer Persönlichkeit einerseits und sozialer Devianz andererseits entgegen.

Für die forensische Anwendung, die sich um Pragmatik und Vermittelbarkeit in foro bemühen muss, wurde deshalb, wenn es um die Frage geht, ob eine Persönlichkeitsstörung mit rechtlicher Erheblichkeit vorliegt, eine gewisse Präzisierung gegenüber den Formulierungen in der herkömmli-

Prof. Dr. med. Henning $\mathrm{Sa} ß$

hsass@ukaachen.de

1 Klinik für Psychiatrie, Psychotherapie und Psychosomatik, Universitätsklinikum der RWTH Aachen, Aachen, Deutschland

2 Klinik für Forensische Psychiatrie, Psychiatrische Universitätsklinik Zürich, Zürich, Schweiz chen Diagnostik und in den gängigen Klassifikationssystemen vorgeschlagen: Danach liegt eine forensisch belangvolle Persönlichkeitsstörung dann vor, wenn durch Ausprägungsgrad und/oder die besondere Konstellation von psychopathologisch relevanten Merkmalen erhebliche subjektive Beschwerden und/oder nachhaltige Beeinträchtigungen der sozialen Anpassung entstehen (Saß 1987). Diese Formulierung geht also durch die Einführung der Qualifizierung ,psychopathologisch relevant“" über die allgemeinen Definitionen von Persönlichkeitsstörung hinaus.

Dabei liegt der wesentliche, auf die forensisch notwendigen Differenzierungen gerichtete Gesichtspunkt darin, dass hartnäckiges sozial abweichendes Verhalten allein nicht ausreicht, um eine rechtlich bedeutsame Persönlichkeitsstörung festzustellen, vielmehr müssen psychopathologisch zu erfassende symptomatologische Beziehungen zu den psychischen Erkrankungen im engeren Sinne bestehen. Anderenfalls wird das Missverständnis gebahnt, dass mit der Vergabe einer Diagnose aus dem reichen Katalog der Klassifikationssysteme bereits eine für Fragen etwa der Schuldfähigkeit oder der Unterbringung im Maßregelvollzug relevante Feststellung getroffen sei. Das ist aber gerade im Gebiet der antisozialen Persönlichkeitsstörungen und der Psychopathie mitnichten der Fall.

Bei einer solchen Konzeption von Persönlichkeitsstörungen mit forensischer Erheblichkeit gehören zu den psychopathologisch relevanten Phänomenen im obigen Sinne etwa dynamische Verschiebungen im Antriebs- und Stimmungsbereich, Auffälligkeiten des Temperaments, der Triebe und der Emotionalität, Affektlabilität, Impulsivität, emotionale Verflachung, Kritikschwäche, Sprunghaftigkeit, Kontaktstörungen oder Besonderheiten des formalen und inhaltlichen Denkens bis hin zu wahnähnlichen Entwicklungen. Dagegen gehören zu den im forensischen Kontext geläufigen, aber ohne begleitende psychopathologische Symptomatik nicht schuldfähigkeitsmindernden Persönlichkeitsei- 
genschaften etwa Halt- und Willensschwäche, Neigung zu Hochstapelei und Betrug, Geltungsbedürfnis, egozentrischunempathische Rücksichtslosigkeit und Gewöhnung an rezidivierende Delinquenz ( $\mathrm{Sa} ß$ 1987, Saß und Habermeyer 2007).

Würde man auf den Gesichtspunkt der psychopathologischen Qualität auffälliger oder störender Persönlichkeitsmerkmale verzichten, bestünde die Gefahr, dass bereits abweichendes, normverletzendes Verhalten allein als Beleg für eine gestörte Persönlichkeit genommen wird. Dies kann gerade in rechtlichem Kontext zu Zirkelschlüssen der Art führen, dass dissoziales, delinquentes Verhalten und die Attribuierung einer Persönlichkeitsstörung wechselseitig als Begründung füreinander dienen. Insofern sind also im forensischen Kontext besondere Vorsichtsmaßregeln beim Umgang mit Persönlichkeitsstörungsdiagnosen geboten, insbesondere im Zusammenhang mit Dissozialität.

Neben solchen konzeptionellen Überlegungen ist auf einige terminologische Besonderheiten im Umfeld der Begriffe Persönlichkeitsstörung und Psychopathie einzugehen. Zunächst könnten sich psychiatriehistorisch interessierte Leser darüber wundern, warum beide Bezeichnungen unterschieden werden, denn vor mittlerweile 95 Jahren hat Schneider in seiner Monographie Die psychopathischen Persönlichkeiten (1923) mit dem Begriff der Psychopathie die Gesamtheit der persönlichkeitsgebundenen Auffälligkeiten belegt, unter denen die Betroffenen, aber auch deren Umgebung leiden. Der mit der Symptomatik verbundene Leidensdruck findet sich bis heute als ein allgemeines Kriterium für die Diagnose einer Persönlichkeitsstörung in den psychiatrischen Klassifikationssystemen DMS-5 und ICD-10, die darüber hinaus dem Ansatz von Schneider in ihrer möglichst wertfreien, deskriptiv-beschreibenden Herangehensweise Rechnung tragen. Die Ausführungen von Schneider können daher als Grundlage für das aktuelle Verständnis von Persönlichkeitsstörungen gelten und der von ihm geprägte Begriff der Psychopathie als Bezeichnung für die Gesamtheit der Persönlichkeitsstörungen. Der Anwendung dieses Begriffes im klinisch-therapeutischen Kontext stand und steht jedoch die damit verbundene abschätzige Bedeutung im allgemeinen Sprachgebrauch entgegen.

Forensisch interessierte Leser wiederum werden mit dem Begriff der Psychopathie vorwiegend die nordamerikanische Begriffsgeschichte bzw. die Entwicklung des Psychopathiekonzepts von Hare (2003) verbinden. Diese fußt auf der Monographie The mask of sanity von Cleckley (1941), die einen besonders rücksichtslosen Persönlichkeitstypus beschrieb. Entsprechend hat auch ein Teil der Autoren dieser Ausgabe in ihren Arbeiten den englischen Begriff ,psychopath" verwendet, um den Unterschied zum Psychopathiebegriff von Schneider zu betonen. Allerdings wurde nach intensiven Diskussionen innerhalb der Autorenschaft der deutschen Ausgabe der PCL-R (Mokros et al. 2017) entschieden, im Manual die Begriffe „Psychopathie“ „Psychopath“ und „psychopathisch“ zu verwenden, weil man davon ausging, dass diese vom Großteil der Anwender ohnehin mit der Hare-Psychopathy gleichgesetzt werden. Entsprechend ist zu erwarten, dass sich im deutschen Sprachraum der Begriff Psychopathie für das Konzept von Hare (2003) durchsetzen wird.

Unabhängig von diesen terminologischen Abgrenzungsschwierigkeiten ist problematisch, dass die Psychopathie sensu Hare mittlerweile oft mit der dissozialen bzw. antisozialen Persönlichkeitsstörung der ICD-10 bzw. des DSM-5 gleichgesetzt wird. Obwohl fast jeder Psychopath im Strafund Maßregelvollzug auch anti- bzw. dissozial ist, ist dies nicht sinnvoll, denn nur ein Teil der Personen mit einer anti- bzw. dissozialen Persönlichkeitsstörung erfüllt auch die Kriterien für Psychopathie (Mokros et al. 2017). Das Konzept von Hare ist deutlich vielschichtiger, als es die Störungskonzepte der ICD-10- und DSM-5-Klassifikation sind, denn es beinhaltet auch narzisstische, impulsive und histrionische Facetten. Insofern erscheint es durchaus angemessen, dass einige der im Heft vertretenen Autoren im Titel ihres Beitrags explizit zwischen Psychopathie und antisozialer Persönlichkeitsstörung unterscheiden.

Vor diesem konzeptionellen und terminologischem Hintergrund beleuchten die Beiträge zum Schwerpunkt „Persönlichkeitsstörung und Psychopathie“ einen breiten Themenkreis: Zu Beginn wird die aktuelle Version der PCL von Hare in ihrer deutschen Fassung durch Hollerbach, Habermeyer, Nitschke und Mokros vorgestellt. Der Beitrag von Herpertz über neurobiologische Grundlagen von Empathie bei Persönlichkeitsstörungen behandelt ein Thema, mit dem zuweilen in forensischem Kontext recht undifferenziert argumentiert wird. Die Zusammenhänge zwischen Sexualdelinquenz und antisozialen oder psychopathischen Persönlichkeitsstörungen werden von Briken und Tozdan dargestellt. Eine Übersicht von Ortner, Preiß und Sevecke fasst die Kenntnisse und Perspektiven bezüglich der jugendlichen Psychopathen, einschließlich der Übergänge zum Erwachsenenalter, zusammen. Die wichtige Frage dimensionaler Ansätze in der Diagnostik von Persönlichkeitsstörungen diskutieren Yoon, Krüppel, Mokros und Zimmermann.

Trotz des in zwei Cochrane-Reviews (Gibbon et al. 2010; Khalifa et al. 2010) konstatierten Mangels an strikt evidenzbasierten Therapiestrategien erscheint es uns sinnvoll, die Diskussion über erfolgversprechende Behandlungsmethoden bei antisozialem Verhalten und auch bei Psychopathie wieder aufzunehmen. So berichtet Fiedler über multimodale Ansätze zur Psychotherapie dissozialer Probanden, wobei sich interessanterweise ein Therapieoptimismus als wichtiger Faktor für Behandlungserfolg erwies. Der Stand der psychodynamisch orientierten Therapieverfahren wird von Dammann referiert. Die von Knörnschild und Jacob vorgestellte Schematherapie, die sich gegenwärtig v.a. in den 
Niederlanden in empirischer Überprüfung befindet, bietet einen interessanten neuen Behandlungsansatz bei antisozialen Persönlichkeitsstörungen. Abschließend berichten Felthous und Stanford aus der Perspektive in den USA über Möglichkeiten der Pharmakotherapie für impulsive Aggression im Kontext von Psychopathie.

Interessenkonflikt H. Saß und E. Habermeyer geben an, dass kein Interessenkonflikt besteht.

\section{Literatur}

Cleckley H (1941) The mask of sanity. Mosby, St. Louis

Gibbon S, Duggan C, Stoffers J, Huband N, Völlm BA, Ferriter M, Lieb K (2010) Psychological interventions for antisocial personality disorder. Cochrane Database Syst Rev 6:CD7668
Hare RD (2003) Manual for the psychopathy checklist - revised, 2. Aufl. Multi Health Systems, Toronto

Khalifa N, Duggan C, Stoffers J, Huband N, Völlm BA, Ferriter M, Lieb K (2010) Pharmacological interventions for antisocial personality disorder. Cochrane Database Syst Rev 8:CD7667

Mokros A, Hollerbach P, Nitschke J, Habermeyer E (2017) PCL-R hare psychopathy checklist revised. Hogrefe, Göttingen

Saß H (1987) Psychopathie - Soziopathie - Dissozialität: Zur Differentialtypologie der Persönlichkeitsstörungen. Springer, Berlin, Heidelberg, New York

$\mathrm{Sa}$ H, Habermeyer E (2007) Die Begutachtung von Persönlichkeitsstörungen aus psychopathologischer Sicht. Forens Psychiatr Psychol Kriminol 1:156-161

Schneider K (1923) Die psychopathischen Persönlichkeiten, 1. Aufl. Deuticke, Wien 